\title{
Programa de entrenamiento en reparacion vascular en modelo experimental para residentes de cirugía general
}

\section{Vascular reparation training program in experimental model for general surgery residents}

Miguel A. Sierra-Juárez", Christian I. Cruz-Romero2*, Ansony R. Godinez-VidaP y

Marco A. Durán-Padilla ${ }^{3}$

${ }^{1}$ Servicio de Angiología y Cirugía Vascular; ${ }^{2}$ Cirugía General; ${ }^{3}$ Servicio de Patología. Hospital General de México Dr. Eduardo Liceaga, Ciudad de México, México

\section{Resumen}

Introducción: Los programas de entrenamiento simulado permiten optimizar recursos, aumentar la práctica técnica y acortar curvas de aprendizaje, y constituyen un método seguro, estandarizado y validado de aprendizaje para todos los entrenados. Objetivo: Desarrollar un programa de entrenamiento vascular mediante la elaboración de una anastomosis término-terminal de un vaso en un modelo experimental para residentes de cirugía general. Método: Para el desarrollo de este modelo de entrenamiento se utilizó una extremidad amputada a nivel supracondíleo libre de proceso médico-legal. Se realizó la disección de la arteria femoral, se dividió en dos porciones y se realizó una anastomosis término-terminal. Se diseño un sistema que incluye la evaluación de las siguientes variables utlizando la escalas OSATS (Objetive Structured Assesment of Technical Skills) y Likert: 1) nudo manual y punto simple; 2) permeabilidad de la anastomosis; 3) simetría de los puntos; 4) fuga de anastomosis; y 5) tiempo total de anastomosis. Discusión: Al reconocer las ventajas del entrenamiento experimental, se propone un modelo biológico experimental reproducible, estandarizado y de bajo costo. Conclusiones: El desarrollo de modelos biológicos experimentales permite al cirujano general la adquisición de habilidades y destrezas quirúrgicas, mejorando el desempeño durante un procedimiento quirúrgico y reduciendo riesgos.

PALABRAS CLAVE: Simulación. Anastomosis. Fuga de anastomosis.

\begin{abstract}
Introduction: Simulated training programs allow to optimize resources, increase technical practice and shorten learning curves, constituting a safe, standardized and validated method of learning for all those trained. Objective: Develop a vascular training program through the elaboration of an end to end anastomosis of a vessel in an experimental model for General Surgery residents. Method: For the development of this training model, an amputated limb was used at the supracondylar level free of medical-legal process. The dissection of the femoral artery was performed, it was divided into two portions and an end to end anastomosis was performed. A system was designed that included the evaluation of the following variables using the OSATS (Objective Structured Assessment of Technical Skills) and Likert scales: 1) manual knot and single stitch; 2) permeability of the anastomosis; 3) symmetry of the stitches; 4) Anastomosis leakage; and 5) total time of the anastomosis. Discussion: Recognizing the advantages of experimental training, a reproducible, standardized, and low cost experimental biological model is proposed. Conclusions: The development of experimental biological models, allows the general surgeon the acquisition of surgical skills and abilities, improving performance during a surgical procedure and reducing risks.
\end{abstract}

KEY WORDS: Simulation training. Anastomosis. Anastomotic leak.

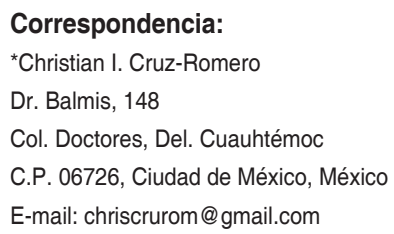

Fecha de recepción: 11-02-2018

Fecha de aceptación: 14-08-2018

DOI: 10.24875/CIRU.18000163
Cir Cir. 2018;86:481-484

Contents available at PubMed www.cirugiaycirujanos.com 


\section{Introducción}

La cirugía se ha utilizado como tratamiento médico a través de los tiempos; sin embargo, la pregunta sobre cómo obtener las habilidades y destrezas quirúrgicas sigue siendo un tema de intenso debate. La educación médica en cirugía general se ha alejado gradualmente del paradigma tradicional de capacitacion de aprendices, siendo una evolución multifactorial que, dentro de sus avances, incluye una mayor estandarización en la capacitación, una supervisión más estricta de las horas de trabajo y un enfoque de competencia y evaluación de las habilidades'.

Siendo el objetivo graduar cirujanos competentes, los educadores quirúrgicos han reconocido la necesidad de mejorar la eficiencia y la efectividad del entrenamiento quirúrgico ${ }^{2}$. La especialidad de cirugía general se cursa durante 4 años en hospitales sede, públicos o privados, del país y comprende tres áreas: 1) cognoscitiva, en la que se fundamentan los procedimientos diagnósticos; 2) psicomotora, que señala las destrezas indispensables para desarrollar en el quirófano; y 3) afectiva, donde se aprenden actitudes que se sostendrán ante el enfermo y sus familiares ${ }^{3}$.

Para que el proceso de enseñanza-aprendizaje sea eficiente, debe centrarse en el aprendizaje significativo, centrado más en el método que en el conocimiento mismo, en el que la figura importante es el alumno y no el maestro; enfatizar más el aprendizaje y no tanto la enseñanza, cuestionar sistemáticamente el conocimiento establecido, romper los estereotipos, propiciar la reflexión crítica más que la memorización, y que el alumno participe en la planeación y en la retroalimentación del programa 4 .

La simulación es la técnica de imitar algún proceso por medio de un equipo que asemeje a la realidad con el propósito de mejorar habilidades y destrezas. En medicina, el uso de simuladores es fundamental por la seguridad del paciente, lo que obliga a nuevos procesos de enseñanza ${ }^{5}$. Jakimowicz ${ }^{6}$ señala la importancia de los modelos de realidad virtual y sus ventajas, como son la evaluación objetiva del desempeño, la interactividad, la disponibilidad de video y la instrucción didáctica. La simulación ha permitido la adquisición de competencias técnicas en cirugía tradicional abierta y laparoscópica en posgrado ${ }^{7}$. Los programas de entrenamiento simulado permiten optimizar recursos, aumentar la práctica técnica y acortar curvas de aprendizaje, y constituyen un modo seguro, estandarizado y validado de aprendizaje para todos los entrenados ${ }^{8,9}$.

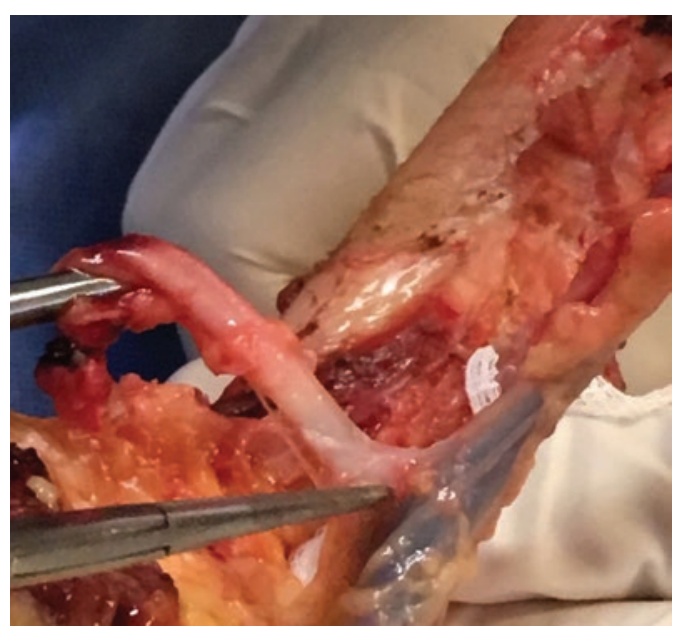

Figura 1. Disección de arteria femoral

El objetivo principal fue desarrollar un programa de entrenamiento vascular mediante la elaboración de una anastomosis término-terminal de un vaso en un modelo experimental. Los objetivos secundarios incluyeron: 1) desarrollar un instrumento para evaluar el desempeño técnico, determinar su confiabilidad y validez en el desarrollo de anastomosis vasculares; y 2) crear un programa de entrenamiento en cirugía vascular para residentes de cirugía general.

\section{Método}

Para el desarrollo de este modelo de entrenamiento se utilizó una extremidad amputada a nivel supracondíleo libre de proceso médico-legal. Se realizó la disección de la arteria femoral, se dividió en dos porciones y se realizó una anastomosis término-terminal. Otros materiales indispensables para esta práctica son porta-agujas, pinza de disección, tijera Metzembaum, sonda de alimentación de $8 \mathrm{Fr}$, sutura no absorbible vascular (polipropileno 6-0), sutura no absorbible (seda 2-0), guantes, gasas y agua inyectable.

El modelo consiste en la disección de la arteria femoral en la extremidad amputada (Fig. 1), realizando una separación cuidadosa hasta lograr una longitud aproximada de $10 \mathrm{~cm}$. Se verifica la permeabilidad del vaso sanguíneo. El siguiente paso es colocar en el extremo proximal la sonda de alimentación, sujetarla con un nudo (seda 2-0), infundir $10 \mathrm{ml}$ de agua inyectable y verificar nuevamente la permeabilidad (Fig. 2). Una vez asegurado el extremo proximal, se realiza un corte transversal total para simular la lesión vascular con tijera Metzembaum (Fig. 3). Se realiza la anastomosis término-terminal con puntos continuos (polipropileno 6-0 vascular), se verifican la permeabilidad y la hermeticidad 


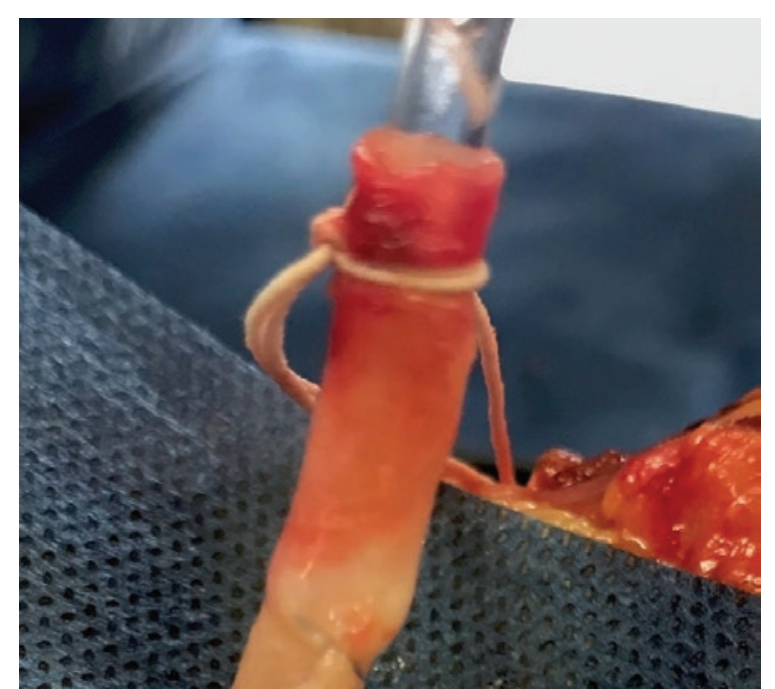

Figura 2. Prueba de permeabilidad vascular con agua inyectable.

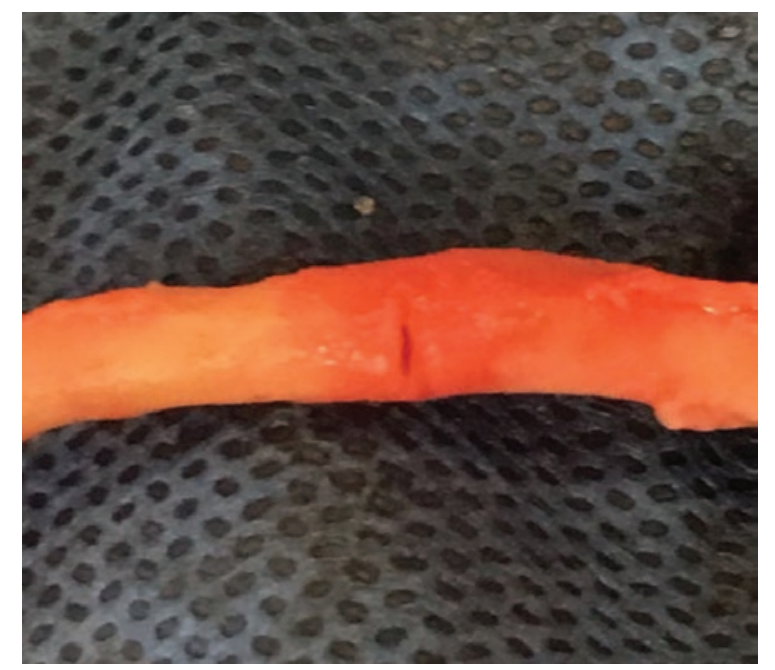

Figura 3.Lesión vascular simulada mediante el corte transversal.

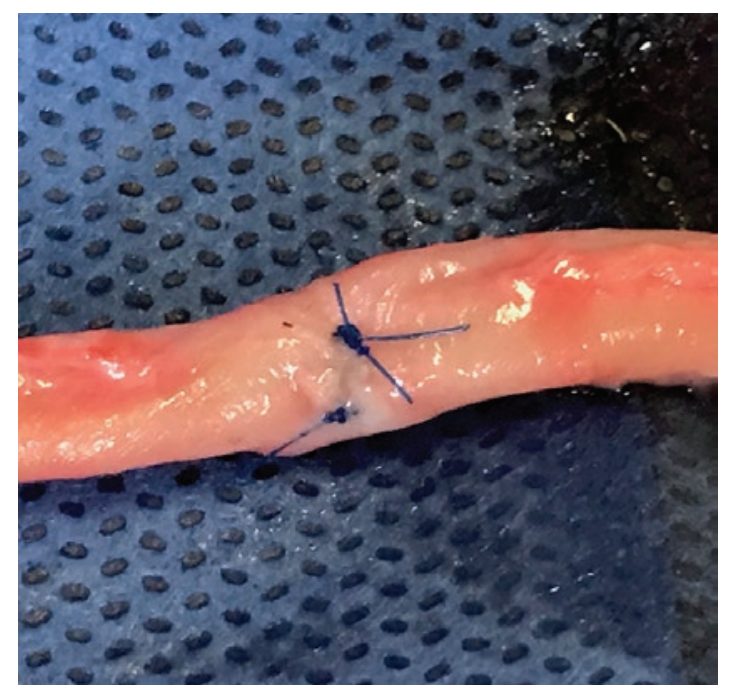

Figura 4. Permeabilidad y hermeticidad de anastomosis términoterminal. de la anastomosis infundiendo a través de la sonda de alimentación $20 \mathrm{ml}$ de agua inyectable (Fig. 4). Por último, se evalúa la cantidad de fuga de anastomosis mediante la instilación de $20 \mathrm{ml}$ de agua inyectable a $4 \mathrm{ml} / \mathrm{s}$, colocando en el extremo distal un recipiente graduado en mililitros y observando la diferencia entre el volumen instilado y el volumen final.

Se diseñó un sistema de evaluación práctica en el que se incluyeron las siguientes variables al realizar la anastomosis término-terminal: 1) nudo manual y punto simple; 2) permeabilidad de la anastomosis; 3) simetría de los puntos; 4) fuga de solución de la anastomosis; y 5) tiempo total de la anastomosis. Se utilizó la escala de evaluación global OSATS (Objetive Structured Assessment of Technical Skills), validada internacionalmente $\left(\right.$ Tabla 1) ${ }^{7}$. Se considera importante medir las actitudes y el grado de conformidad del evaluador, incluyendo en nuestro sistema de evaluación los cinco puntos de la escala de Likert: 1) totalmente en desacuerdo; 2) en desacuerdo; 3) ni de acuerdo ni en desacuerdo; 4) de acuerdo; y 5) totalmente de acuerdo.

\section{Discusión}

El entrenamiento de residentes de cirugía general se ha realizado mediante demostraciones en vivo, siempre dirigido por cirujanos experimentados y calificados. El uso de modelos experimentales ha demostrado incrementar las habilidades quirúrgicas, con una disminución de riesgos y una optimización del tiempo quirúrgico. Al reconocer las ventajas del entrenamiento experimental, se propone un modelo biológico experimental reproducible, estandarizado y de bajo costo.

La adquisición de habilidades y destrezas para lograr cirujanos competentes suele ser heterogénea, dado que no se cuenta con un método objetivo para definir un nivel de habilidad, por lo que el uso de modelos y simuladores es de gran utilidad para evaluar la capacidad de desempeño de un procedimiento quirúrgico ${ }^{10}$.

Se ha reportado en la literatura que los simuladores son efectivos durante la fase inicial de entrenamiento, y han sido validados por diversos estudios ${ }^{1,2}$. El uso de modelos biológicos sigue siendo aceptable para explorar y mejorar técnicas quirúrgicas sin comprometer la seguridad del paciente ${ }^{11}$. La principal limitación que presentan los modelos biológicos de cadáver es la falta de una hemorragia inminente, pero son de gran utilidad en la adquisión inicial de habilidades cognitivas y 
Tabla 1. Escala OSATS (Objetive Structured Assessment of Technical Skills) de evaluación de habilidades generales

\begin{tabular}{|c|c|c|c|c|c|}
\hline Puntuación & Respeto por los tejidos & $\begin{array}{l}\text { Tiempo y } \\
\text { movimientos }\end{array}$ & Uso del instrumento & $\begin{array}{l}\text { Flujo de la cirugía y } \\
\text { planificación posterior }\end{array}$ & Conocimiento \\
\hline 1 & $\begin{array}{l}\text { Uso frecuente de fuerza } \\
\text { innecesaria } \\
\text { Daño causado por uso } \\
\text { inapropiado de los } \\
\text { instrumentos }\end{array}$ & $\begin{array}{l}\text { Muchos movimientos } \\
\text { innecesarios }\end{array}$ & $\begin{array}{l}\text { Repetidamente hace } \\
\text { movimientos vacilantes }\end{array}$ & $\begin{array}{l}\text { Frecuentemente detiene } \\
\text { el procedimiento o } \\
\text { requiere discutir los pasos } \\
\text { siguientes }\end{array}$ & $\begin{array}{l}\text { Conocimiento } \\
\text { deficiente y requiere } \\
\text { instrucciones en la } \\
\text { mayoría de los pasos } \\
\text { quirúrgicos }\end{array}$ \\
\hline \multicolumn{6}{|l|}{2} \\
\hline 3 & $\begin{array}{l}\text { Manejo cuidadoso } \\
\text { de los tejidos, pero } \\
\text { ocasionalmente produce } \\
\text { daño inadvertido }\end{array}$ & $\begin{array}{l}\text { Relación } \\
\text { entre tiempo y } \\
\text { movimientos } \\
\text { eficiente, pero } \\
\text { realiza algunos } \\
\text { movimientos } \\
\text { innecesarios }\end{array}$ & $\begin{array}{l}\text { Uso competente de } \\
\text { los instrumentos, pero } \\
\text { ocasionalmente se } \\
\text { observa rígido o torpe }\end{array}$ & $\begin{array}{l}\text { Demuestra habilidades } \\
\text { para planear los } \\
\text { pasos siguientes, con } \\
\text { progresión constante del } \\
\text { procedimiento }\end{array}$ & $\begin{array}{l}\text { Conoce todos los } \\
\text { aspectos importantes } \\
\text { de la operación }\end{array}$ \\
\hline \multicolumn{6}{|l|}{4} \\
\hline 5 & $\begin{array}{l}\text { Maneja los tejidos } \\
\text { apropiadamente con } \\
\text { mínimo daño }\end{array}$ & $\begin{array}{l}\text { Economía de } \\
\text { movimientos } \\
\text { y máxima } \\
\text { eficiencia }\end{array}$ & $\begin{array}{l}\text { Movimientos fluidos } \\
\text { con los instrumentos y } \\
\text { sin torpeza }\end{array}$ & $\begin{array}{l}\text { Curso planeado de la } \\
\text { operación en forma obvia, } \\
\text { con flujo sin esfuerzo }\end{array}$ & $\begin{array}{l}\text { Familiaridad } \\
\text { demostrada con } \\
\text { todos los aspectos de } \\
\text { la operación }\end{array}$ \\
\hline
\end{tabular}

Fuente: modificada de Martin, et al. ${ }^{7}$

hápticas que, sin duda, proporcionan un beneficio al realizar un procedimiento quirúrgico ${ }^{12}$.

\section{Conclusiones}

El desarrollo de modelos biológicos experimentales permite al cirujano general la adquisición de habilidades y destrezas quirúrgicas, mejorando el desempeño durante un procedimiento quirúrgico y reduciendo riesgos. Este programa simulado plantea un método alternativo y reproducible para el desarrollo de competencias técnicas, y puede ser incorporado al programa para la formación de cirujanos generales.

\section{Responsabilidades éticas}

Protección de personas y animales. Los autores declaran que los procedimientos seguidos se conformaron a las normas éticas del comité de experimentación humana responsable y de acuerdo con la Asociación Médica Mundial y la Declaración de Helsinki.

Confidencialidad de los datos. Los autores declaran que en este artículo no aparecen datos de pacientes.

Derecho a la privacidad y consentimiento informado. Los autores declaran que en este artículo no aparecen datos de pacientes.

\section{Conflicto de intereses}

Los autores declaran no tener ningún conflicto de intereses.

\section{Bibliografía}

1. Ghaderi I, Fitzgibbons S, Watanabe Y, Lachapalle A, Piage J; Association for Surgical Education Simulation Committee. Surgical skill curricula in American College of Surgeon accredited education institutes: an international survey. Am J Surg. 2016;213:678-86.

2. Badash I, Burtt K, Solorzano C, Carey J. Innovations in surgery simulation: a review of past, current and future techniques. Ann Transl Med. 2016;4:453

3. Córdoba D, Ojeda G, Sapién J, Rosas BJ, Morales MP. La especialidad de cirugía general: experiencias de residentes. Rev Espec Médico-Quirúrgicas. 2009;14(2):79-82.

4. Vega J, Zaldívar G, Ávila J, Vega J, Romero J. El cirujano científico y la enseñanza de la cirugía en México. Segunda parte: La enseñanza de la cirugía en el postgrado y educación médica continua del cirujano. Cirujano General. 2004;26(4):330-6.

5. Carrasco Rojas JA, García CB, Carrasco JA. Utilización de simuladores en la educación quirúrgica. Cirujano General. 2013;35(Supl 1):S62-5.

6. Jakimowicz J. Simulación en cirugía, ¿dónde estamos y a dónde llegaremos? Cir Cir 2011;79:44-49.

7. Martin JA, Regehr G, Reznick R, MacRae H, Mur-naghan J, Hutchison C, et al. Objective structured assessment of technical skill (OSATS) for surgical residents. Br J Surg. 1997;84:273-8

8. Van Nortwick SS, Lendvay TS, Jensen AR, Wright AS, Horvath KD, Kim S. Methodologies for establishing validity in surgical simulation studies. Surgery. 2010;147:622-30.

9. Reznick RK, MacRae H. Teaching surgical skills - changes in the wind. N Engl J Med. 2006;355:2664-9.

10. Gallagher AG. Metric-based simulation training to proficiency in medical education: what it is and how to do it. Ulster Med J. 2012;81:107-13.

11. Shamin K, Ahmed K, Gavazzi A. Development and implementation of centralized simulation training: evaluation of feasibility, acceptability and construct validity. BJU Int. 2013;111:518-23.

12. Sutton E, Billeter A, Druen D, Roberts H, Rice J. Development of a human cadaver model for training in laparoscopic donor nephrectomy. Clin Transplant. 2017;31(6). doi: 10.1111/ctr.12979. Epub 2017 Apr 24. 